I dent i f i cat i on of novel suppressors for Mog1 i mpl i es its i nvol vement i $n$ RNA met abol i sm, I i pi d met abol i smand si gnal t $r$ ansduct i on

\begin{tabular}{|c|c|}
\hline 著者 & $\begin{array}{ll}\text { OK Nasaya, MA Li, WANG Yonggang, HATANAKA } \\
\text { Aki ra, M YAZATO Chi e, TATEBAYASH } & \text { Kazuo, } \\
\text { N SH TAN H deo, UCH DA Hi r oyuki, N SH MOTO } \\
\text { Takehar u }\end{array}$ \\
\hline $\begin{array}{l}\text { j our nal or } \\
\text { publ i cat i on title }\end{array}$ & Gene \\
\hline vol une & 400 \\
\hline nunber & 1- 2 \\
\hline page $r$ ange & 114121 \\
\hline year & $2007-10$ \\
\hline URL & ht t p: //hdl . handl e. net /10098/1212 \\
\hline
\end{tabular}




\title{
Identification of novel suppressors for Mog1 implies its involvement in RNA metabolism, lipid metabolism and signal transduction
}

\author{
Masaya Oki ${ }^{\mathrm{a}, \mathrm{c}}$, Li Ma ${ }^{\mathrm{a}, 1}$, Yonggang Wang ${ }^{\mathrm{a}, 2}$, Akira Hatanaka ${ }^{\mathrm{c}}$, Chie Miyazato ${ }^{\mathrm{b}}$, \\ Kazuo Tatebayashi ${ }^{b}$, Hideo Nishitani ${ }^{a, 3}$, Hiroyuki Uchida ${ }^{c}$, Takeharu Nishimoto ${ }^{a, *}$ \\ "Department of Molecular Biology, Graduate School of Medical Science, Kyushu University, Fukuoka 812-8582, Japan \\ b Division of Moleculor Cell Signaling, Institute of Medical Science, The University of Tokyo, 4-6-I Shirokanedai, Minato-ku,Tokyo 108-8639, Japan \\ " Department of Applied Chemistry \& Biotechnology, Faculty of Engineering. University of Fukui, Fukui 910-8507, Japan
}

\begin{abstract}
Mog1 is conserved from yeast to manmal, but its function is obscure. We isolated yeast genes that rescued a temperature-sensitive death of $S$. cerevisiae $S c m o g 1 \Delta$, and of $S$. pombe $S p m o g I^{t s}$. $S c m o g l \Delta$ was rescued by Opi3p, a phospholipid $N$-methyltransferase, in addition to $S$. cerevisiae Ran-homologue Gsplp, and a RanGDP binding protein Ntf2p. On the other hand, Spmog $1^{\text {ts }}$ was rescued by Cid13 that is a poly (A) polymerase specific for suc22 $2^{+}$mRNA encoding a subunit of ribonucleotide reductase, Sspl that is a protein kinase involved in stress response pathway, and Crp79 that is required for mRNA export, in addition to Spil, S. pombe Ran-homologue, and Nxt2, $S$. pombe homologue of Ntf2p. Consistent with the identification of those suppressors, lack of ScMoglp dislocates Opi3p from the nuclear membrane and all of Spmog $I^{\text {ts }}$ showed the nuclear accumulation of mRNA. Furthermore, SpMog1 was co-precipitated with Nxt2 and Cid13.
\end{abstract}

Keywords: Cid13, Crp79, Ntf2p/Nxt2, Ran, Ssp1

\section{Introduction}

Ran GTPase mediates nucleoplasmic transport, microtubule assembly and nuclear membrane formation (Dasso, 2002; Weis, 2003; Mattaj, 2004). Guanine nucleotide triphophate (GTP) of Ran is hydrolized to guanine diphosphate (GDP) by the aid of

Abbreviations: ts, temperaturc-sensitive; Mog1, multicopy suppressor of ts gsp 1; Opi3, overproducer of inositol; NTF2, nuclear transport factor; GST, glutathione $S$-transferase; DAPI, 4'6-diamidino-2-phenylindole.

* Corresponding author. Tel.: +8192642 6175; fax; +81926426183

E-mail address: thishi@molbiol.med.kyushu-u.ac.jp (T. Nishimoto).

1 Division of Hematology, Center for Biologics Evaluation and Research, Food and Drug Administration, Room 305, 29 Lincoln Drive, Bethesda, MD20892, USA.

${ }^{2}$ Laboratory of Gene Regulation and Development, NICHD, NIH, Building 18, Room 106, Bethesda, MD 20892, USA.

${ }^{3}$ Laboratory of Biological Signaling, Graduate School of Life Science, University of Hyogo. Kouto 3-2-1, Kamigori, Ako-gun, Hyogo,678-1297. Japan.
RanGAP/Rna1, Ran GTPase activating protein. Ran-GDP is exchanged to Ran-GTP by RanGEF/RCC1, guanine nucleotide exchange factor. While RanGAP is mainly localized in the cytoplasm, RanGEF/RCCl is localized in the nuclei, creating a gradient of Ran-GTP concentration from the nuclei to the cytoplasm that is important to determine the destination of nucleocytoplasmic transport. Proteins with a nuclear localization signal bind nuclear import receptors in the cytoplasm. Those complexes enter nucleus through the nuclear pore complexes (NPC) (Weis, 2003). In the nucleus, Ran-GTP binds nuclear import receptors, releasing cargo-proteins from importcomplexes. On the other hand, proteins with a nuclear exportsignal are transported to the cytoplasm by the export receptors that bind Ran-GTP. In the cytoplasm, GTP of exported RanGTP is hydrolysed by the aide of RanGAP, terminating the nuclear export. Resulting Ran-GDP is imported to the nucleus by NTF2, nuclear transport factor No. 2 (Ribbeck et al., 1998; Smith et al., 1998). In the nucleus, GDP of Ran-GDP is exchanged 
Table 1

Suppressor genes for Scmogl 14 and Spmog $I^{\text {ts }}$

\begin{tabular}{|c|c|}
\hline Suppressor genes & Frequency* \\
\hline \multicolumn{2}{|l|}{ For Scmog 11} \\
\hline$M O G 1$ & 65 \\
\hline GSPI & 7 \\
\hline NTF2 & 2 \\
\hline$G S P 2$ & 6 \\
\hline$O P I 3$ & 19 \\
\hline \multicolumn{2}{|l|}{ For Spmogl $l^{t s}$} \\
\hline $\operatorname{mog} l^{+}$ & 19 \\
\hline $\operatorname{spil}^{+}$ & 56 \\
\hline$n x t 2^{+}$ & 17 \\
\hline $\operatorname{cid} 13^{+}$ & 2.0 \\
\hline $\operatorname{ssp} 1^{+}$ & 1.6 \\
\hline $\operatorname{crp} 79^{+}$ & 1,0 \\
\hline$S P B C 354.10^{\circ}$ & 3.2 \\
\hline
\end{tabular}

* Ratio $(\%)$ for the total number of independently isolated suppressor genes.

with GTP, releasing NTF2 that will be backed to the cytoplasm (Stewart, 2000). NTF2, an essential protein for nuclear protein import (Moore and Blobel, 1994; Paschal and Gerace, 1995), is thought to function solely for nuclear import of Ran-GDP. In this regard, NTF2 that specifically binds RanGDP is argued to be a Ran-GDP dissociation inhibitor (GDI) (Yamada et al., 2004).

Mog1 was first identified as a suppressor that rescues a temperature-sensitive defect of $S$, cerevisiae Ran, Gsplp, gspl ${ }^{\text {ts }}$ that can be also rescued by $S$. cerevisiae NTF2, Ntf2p (Oki and Nishimoto, 1998). Later on, Mogl is found to be well conserved from yeast to human. Both ScMoglp and mammalian Mog1 bind Ran-GTP. They showed the activity of GTP releasing, but not, that of nucletide exchanging of RanGTP (Steggerda and Paschal, 2000; Oki and Nishimoto, 2000). In this regard, Mog1 is suggested to enhance the GDP $\rightarrow$ GTP exchange by collaborating with RanGEF/RCC1 and RanBP1 (Nicolas et al., 2001), accumulating Ran-GTP in the nucleus to accelerate the nucleocytoplasmic transport. Consistently, when overexpressed, ScMog1p is localized in the nucleus of both yeast and mammals (Oki and Nishimoto, 1998; Tatebayashi et al., 2001; Marfatia et al., 2001) and lack of Mog1p causes nuclear import defect (Oki and Nishimoto, 1998). In S. pombe, however, a nuclear import defect is suggested due to a loss of nuclear envelope integrity that happens in Spmog $1-I^{\text {ts }}$ (Tatebayashi et al., 2001). On the other hand, ScMoglp is reported to be involved in $S$. cerevisiae $S L N 1-S K N 7$ signal transduction ( $\mathrm{Lu}$ et al., 2004), indicating that $\mathrm{Mog} 1 \mathrm{p}$ may function not only in the nucleus, but also in the cytoplasm. Unexpectedly, the crystal structure of $\mathrm{ScMog} 1 \mathrm{p}$ is reported to be similar to PsbP, a membrane-extrinsic subunit of photosystem II from N. tabacum, the hypothetical protein Pa94 from $P$. aeruginosa and TM1622, a hypothetical protein from Thermotoga maritime (Ifuku et al., 2004; Xu et al., 2006). These curious reports force to clarify a biological function of Mog1. Presently we isolated suppressors that rescued a defect of Mog1 using both $S$. cerevisiae and $S$. pombe. In both cases, $\mathrm{Ntf2p}$ and $S$. pombe NTF2-homologue, Ntx2 were obtained as a suppressor. While a major suppressor for Spmog $I^{\text {ts }}$ was Ntx2, the phospholipids $N$-methyltransferase Opi3p mainly suppressed Sc mogl 1 .

\section{Materials and methods}

\subsection{Yeast media and strains}

S. cerevisiae Scmog1 1 (MOY1:MATa mog 1 A::HIS3, ade2101, leu2-Al, trpl-463, ura3-52, lys2-801, his3-4200) (Oki and Nishimoto, 1998) was used to isolate suppressors for loss of
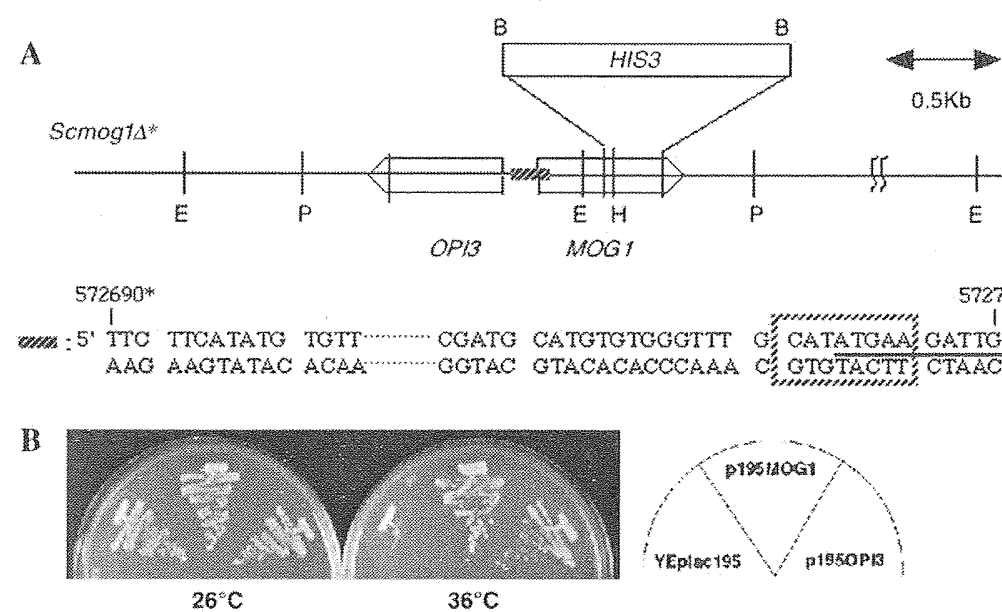

Fig. 1. OPI3 rescued a temperature-sensitive lethality of Scmogl $\Delta^{*}$. A. Construction of Scmog $14^{*}$. The plasmid, pHIS3MOGIN (Oki and Nishimoto, 1998) digested with $X b a$ and $X h o l$ was introduced into YPH499 (Oki and Nishimoto, 1998), resulting in Scmogl $4^{*}$. Nucleotide sequence of the oblique striped region was shown: the squared UAS of OPI3 overlaps with the ORF of Moglp (underlined). *: The nucleotide number on $S$. cerevisiae chromosome X. B. The indicated plasmids,

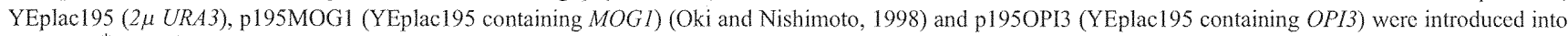
Scmog $1 \Delta^{*}$. URA transformants were incubated on a synthetic medium lacking uracil at the indicated temperature. 
A
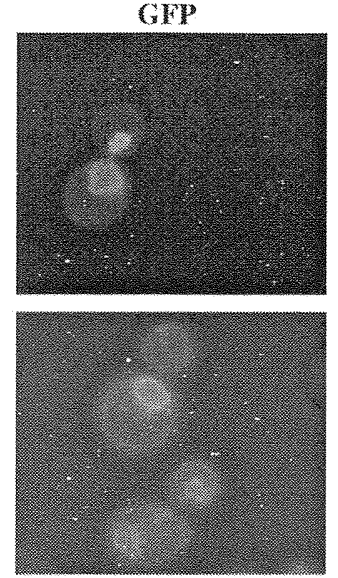

$B$
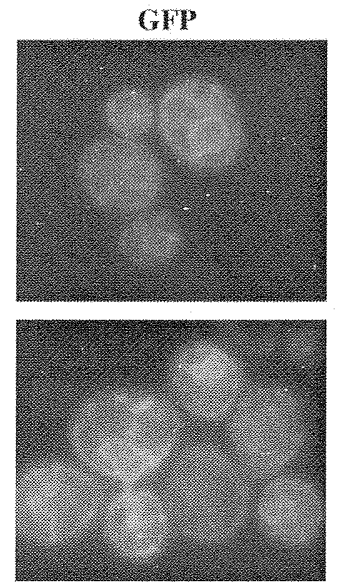

DAPI
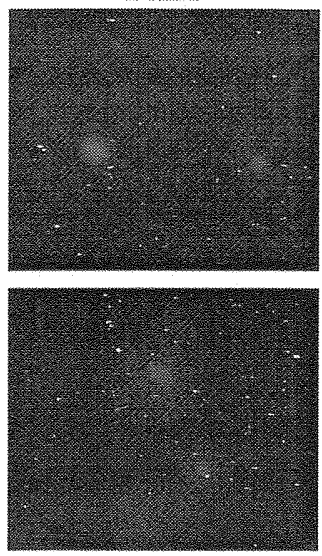

DAPI
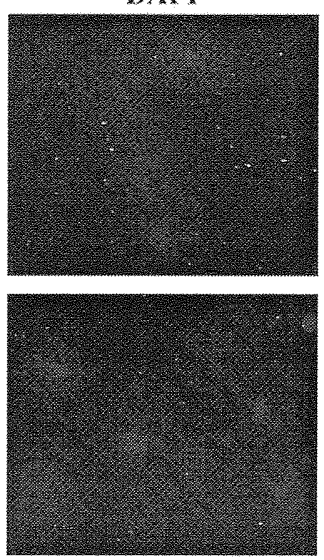

Phase
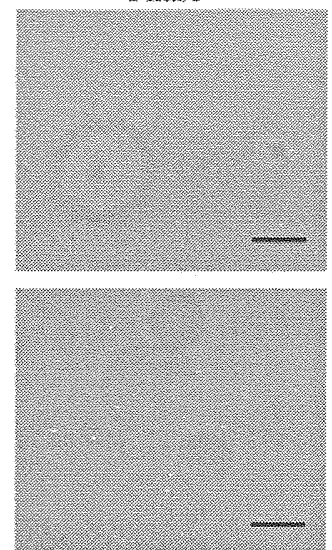

Thase
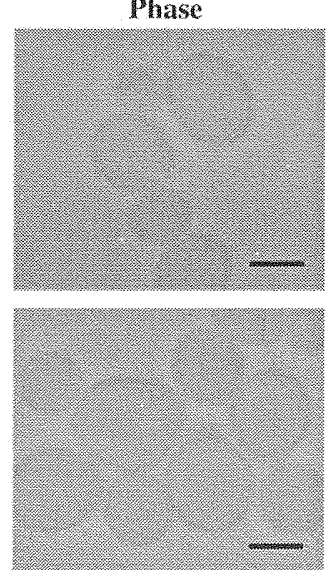

Fig. 2. Localization of OPI3p. Exponentially growing S. cerevisiae MOY11 [p314EGFP-OPI3] (A) and S. cerevisiae MOY12 [p314EGFP-OPI3] (B) were fixed with paraformaldehyde. GFP was observed by fluorescence microscopy. DNA was stained with DAPI. Representative figures are shown. Bar: $2 \mu$ m.

ScMoglp. S. pombe diploid strain $\left(h^{+} / h^{-}\right.$ade6-M216/ade6M210, leul-32/leul-32, ura4-418/ura4- 118 , his7-366/his7$366, \mathrm{mog}^{+} / \operatorname{mog} 1: \therefore \mathrm{ura}^{+}{ }^{+}$) (Tatebayashi et al., 2001) was used to isolate $S$. pombe mog $I^{\text {ts }}$ that was then used for suppressorisolation. $S$. cerevisiae and $S$. pombe strains were grown in medium with appropriate supplements as described by Oki and Nishimoto (1998), and Kusano et al. (2004), respectively. The following new $S$. cerevisiae strains were constructed for this study: MOY11 (MATa opi3 A::HIS3, ade2-101, leu2- 1 , trp 1-463, ura3-52, lys2-801, his3-4200), MOY12 (MATa

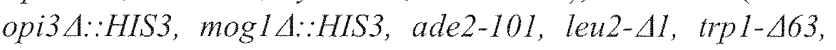
ura3-52, lys 2-801, his3-4200).

\subsection{Isolation of Spmog $1^{\text {ts }}$}

Spmog $1^{+}$genomic DNA was amplified by PCR using following primers: $5^{\prime}$ primer, AAA CTC GAG ATG GTA CAG CTA TTC GGT GGG GCT and 3' primer, CGC GGA TCC GCC TAT GTC CAT CAT CCT TTA AGC. Amplified DNA fragments of 0.6 kilobase pair $(\mathrm{kb})$ were inserted into a vector, pREP81X (Maundrell, 1993). Resulting plasmid, pREP81X-
Spmogl was introduced into S. pombe diploid strain (mogl / $\operatorname{mog} 1::$ ura $\left.4^{+}\right)$to isolate $S$. pombe haploid strain, Spmogl:: ura $^{+}$[pREP81X-Spmog1]. To introduce the mutation, the Spmog $1^{+}$genomic DNA fragment was amplified in the presence of an appropriate concentration of $\mathrm{Mn}^{2+}$, using primer sets: 5' primer, GGT TGA ATA CTT ATT ATC TAG TGA ACG and $3^{\prime}$ primer, TCT TCT TCG TAT GAT GAT ATC CT, as described (Hirose et al., 2006), and then transfered into Spmog 1::ura4 ${ }^{+}$[pREP81X-Spmog1] through electroporation. Transfected cells papillated on plates containing adenine,

Table 2

$\operatorname{SpMog} 1^{\text {ts }}$ and its mutation site

\begin{tabular}{ll}
\hline Spmog $l^{t .5}$ & Amino acid change \\
Spmog $1-2^{t s}$ & S180P \\
Spmog $1-3^{t s}$ & W79R \\
Spmog $l-4^{t s}$ & L9H \\
Spmog $1-5^{t s}$ & F5L \\
Spmog $1-6^{t s}$ & F5S \\
\hline
\end{tabular}

${ }^{1}$ Spmog $1-I^{\mathrm{ts}}$ was reported by Tatebayashi et al., 2001.

${ }^{2}$ Amino acid was shown by a single letter. 

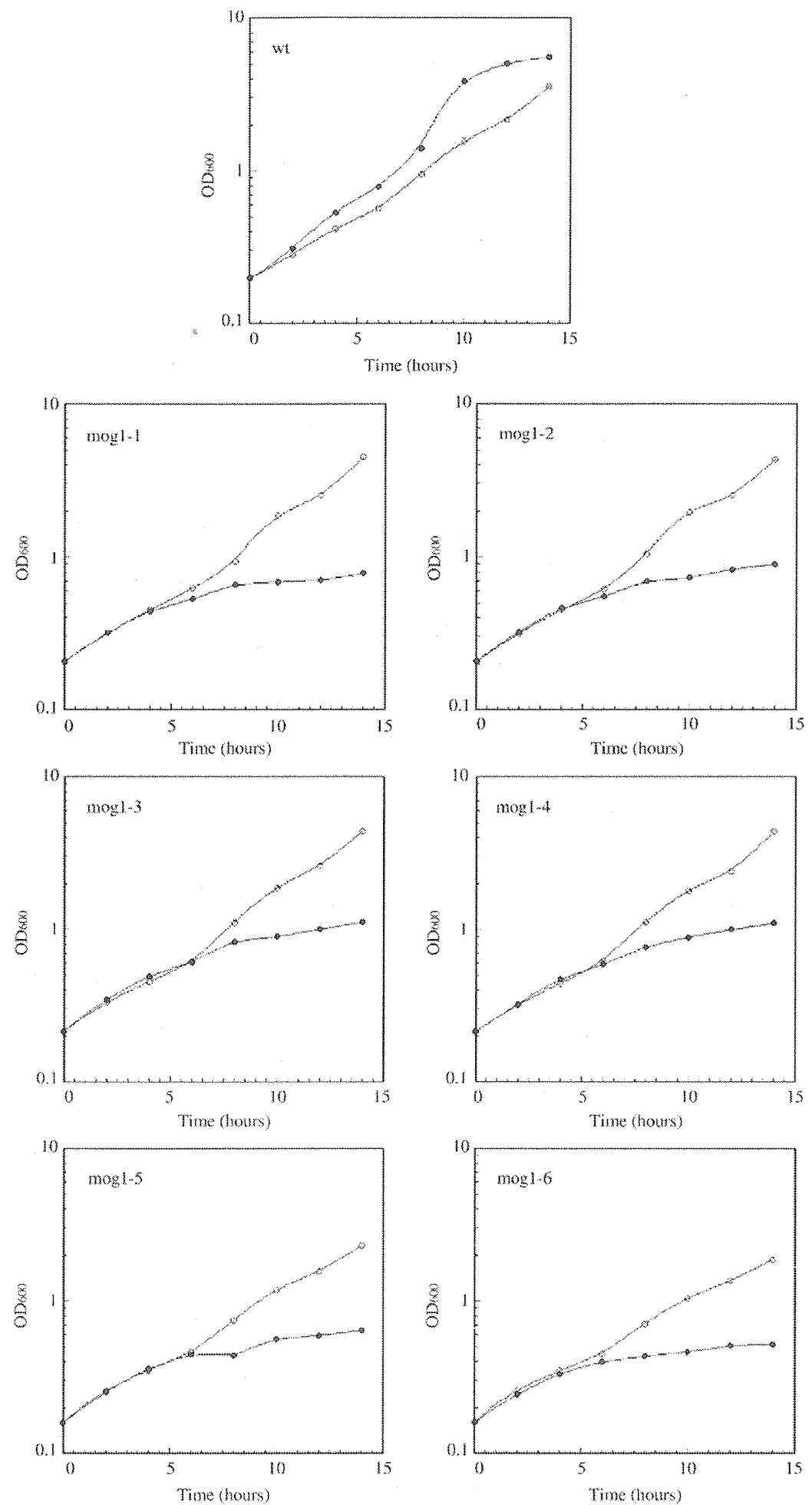

Fig. 3. Growth curve of Spmog $I^{15}$. Overnight cultures of Spmog $I^{\mathrm{wt}}$ and $S p m o g I^{\mathrm{ts}}$ strains were diluted into YES medium and grown at $26^{\circ} \mathrm{C}$ until $\mathrm{OD}$ (000 um $=0.2$. Half of each culture was then shifted to $37^{\circ} \mathrm{C}$ (filled circles) and the other half was further incubated at $26{ }^{\circ} \mathrm{C}$ (open circle).

histidine, uracil, leucine, thiamine and 5-fluoroorotic acid (5-FOA) at $26^{\circ} \mathrm{C}$, were replica-plated on YE5S phloxinB plates, and then incubated at $26^{\circ} \mathrm{C}$ or $36{ }^{\circ} \mathrm{C}$. Temperature sensitive strains were backcrossed with $\operatorname{Spmog} I^{+}$.

\subsection{Isolation of multicopy suppressors}

For Scmog 1 A: the genomic library (RB236) constructed by using the vector YEp24 (Carlson and Botstein, 1982), was 
introduced into cultures of Scmog1 1 . Plasmids were isolated from $t s^{+} U R A^{++}$transformants using $E$. coli as described (Oki and Nishimoto, 1998). S. cerevisiae genomic DNA of recovered plasmid DNA was sequenced to search for an open reading frame (ORF) using the MIPS program (Munich Information Center for Protein Sequence). The $O R F$ S carried on $S$. cerevisiae genomic DNA fragments were inserted into YEplac195 to introduce into Scmogl 1.

For $S p m o g I^{\text {ts }}: S$. pombe genomic library constructed by using the vector pAL-SK, was kindly provided from Dr. M. Yanagida (Kyoto University) and introduced into both Spmog I$3^{\text {ts }}$ and Spmogl-4 $4^{\text {ts }}$ (Table 1) with electroporation. S. pombe genomic DNA recovered from $t s^{+}$transformants as described (Kusano et al., 2004), was sequenced. All ORFs found in recovered $S$. pombe genomic DNA fragments were subcloned into the vector $\mathrm{pAL}-\mathrm{SK}$ and then introduced into Spmog $1^{\mathrm{ts}}$.

\section{4. mRNA export assay}

The mRNA export assay was performed as described (Tani et al., 1996).

\section{Results}

\subsection{OPI3 rescued ScmoglA}

As reported (Oki and Nishimoto, 1998), S. cerevisiae Ran, Gsplp and Gsp2p (Belhumer et al., 1993), and Ntf2p (Paschal and Gerace, 1995), rescued a temperature sensitive death of Scmog1 1 lacks ScMOGl, indicating the genetic system to isolate suppressor genes of $M O G 1$ is working for $S \mathrm{cmog} 1 \triangle$. Opi3p that is the phospholipids $N$-methyltransferase (Greenberg et al., 1982; Boumann et al., 2004) was obtained as a major suppressor for Scmog 1 (Table 1). S. cerevisiae genomic DNA sequence revealed the upstream activation sequence (UAS) of OPI3 (Kodaki et al., 1991) overlaps with ScMOGl-ORF as shown in Fig. 1A, so that it was deleted in Scmogld. Since UAS is essential for an efficient gene expression, we constructed a new null mutant of ScMOGl, Scmogl $\Delta^{*}$ (MOY7: MATa mog1 1::HIS3, ade2-101, leu2-A1, trp1-463, ura3-52, lys 2-801, his3-4200), that lacks the C-terminal region of ScMOG1, but possess the UAS sequence of OPI3 (Fig. 1A). Opi3p also rescued a temperature-sensitive death of $S \mathrm{cmog} l \triangle^{*}$ (Fig. 1B), indicating it is a novel suppressor of $S \mathrm{cmog} / \triangle$. When overexpressed, Opi3p was localized on the periphery of S. serevisiae nucleus (Fig. 2A). Interestingly, Opi3p was dislocated from the nuclear membrane in the absence of ScMog1p (Fig. 2B).

\subsection{Suppressors for Spmog $1^{\text {ts }}$}

Proteins directly interacting with Moglp may not be obtained as a suppressor of Scmogl $\Delta$ that lacks ScMoglp. In order to overcome this issue, we took the advantage of $S$. pombe that $S p m o g I^{+}$is essential for cell-survival (Tatebayashi et al., 2001). A series of temperature-sensitive mutants of Spmog ${ }^{+}$ were isolated using the error prone PCR as described (Oki et al., 1998). Among obtained mutants, five Spmogl $1^{\text {ts }}$ strains that possess a single amino acid change, were chosen for a further analysis (Table 2). Their growth curves were shown in Fig. 3. S. pombe genomic libraries were introduced into Spmog $1-3^{\text {ts }}$ and Spmog $1-4^{\text {ts }}$. Both $s p i 1^{+}$and $n x t 2^{+}$were isolated as suppressor genes of $\operatorname{Spmog} I^{\text {ts }}$ with high frequencies (Table 1). Spil is $S$. pombe Ran homologue (Matsumoto and Beach, 1991) and Nxt2, NTF2-related export protein 2, is $69 \%$ identical to Ntf2p (Black et al., 1999; BLAST Alignment for $S$. cerevisiae NTF2 vs $S$. pombe HITS). Additionally, a temperature-sensitive growth of $\operatorname{Spmog} 1^{\text {ts }}$ was rescued by Cid13, poly (A) polymerase for $s u c 22^{+}$mRNA (Saitoh et al., 2002); Crp79, mRNA export carrier (Thakurta et al., 2002); Ssp1, a protein kinase involved in alteration of cell polarity (Matsusaka et al., 1995; Rupes et al., 1999) and the ORF: SPBC354.10 that is a hypothetical protein (Table 1). A representative result of suppression was shown in Fig. 4. There was no chol $^{+}$, encoding a $S$. pombe homologue of $S$. cerevisiae Opi3p (Kanipes et al., 1998a,b) (Table 1).

\section{3. mRNA export defect of Spmog $1^{\text {ts }}$}

Both Cid13 and Crp79 suggest an involvement of Mog1 in mRNA metabolism. Previous reports, however, are controversial.

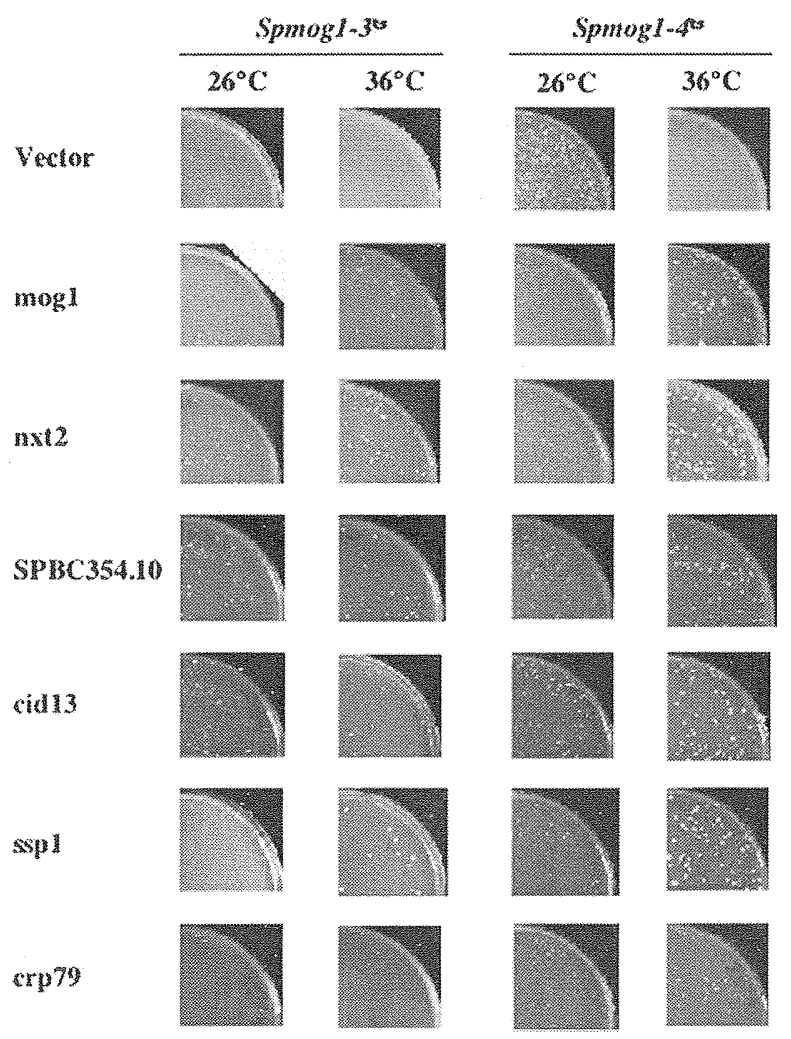

Fig. 4. A temperature sensitive lethality of $\operatorname{Spmog} I^{\text {ts }}$ was rescued by isolated suppressors. The vector, $\mathrm{pAL}-\mathrm{SK}$, and the indicated suppressor genes containing their own promoters carried on pAL-SK were introduced into Spmog $1-3^{\text {ts }}$ and Spmog $1-4^{\text {ts }}$, respectively. Resulting transformants were incubated on YE5S plates supplemented with His, Ade and Ura at the indicated temperature. 


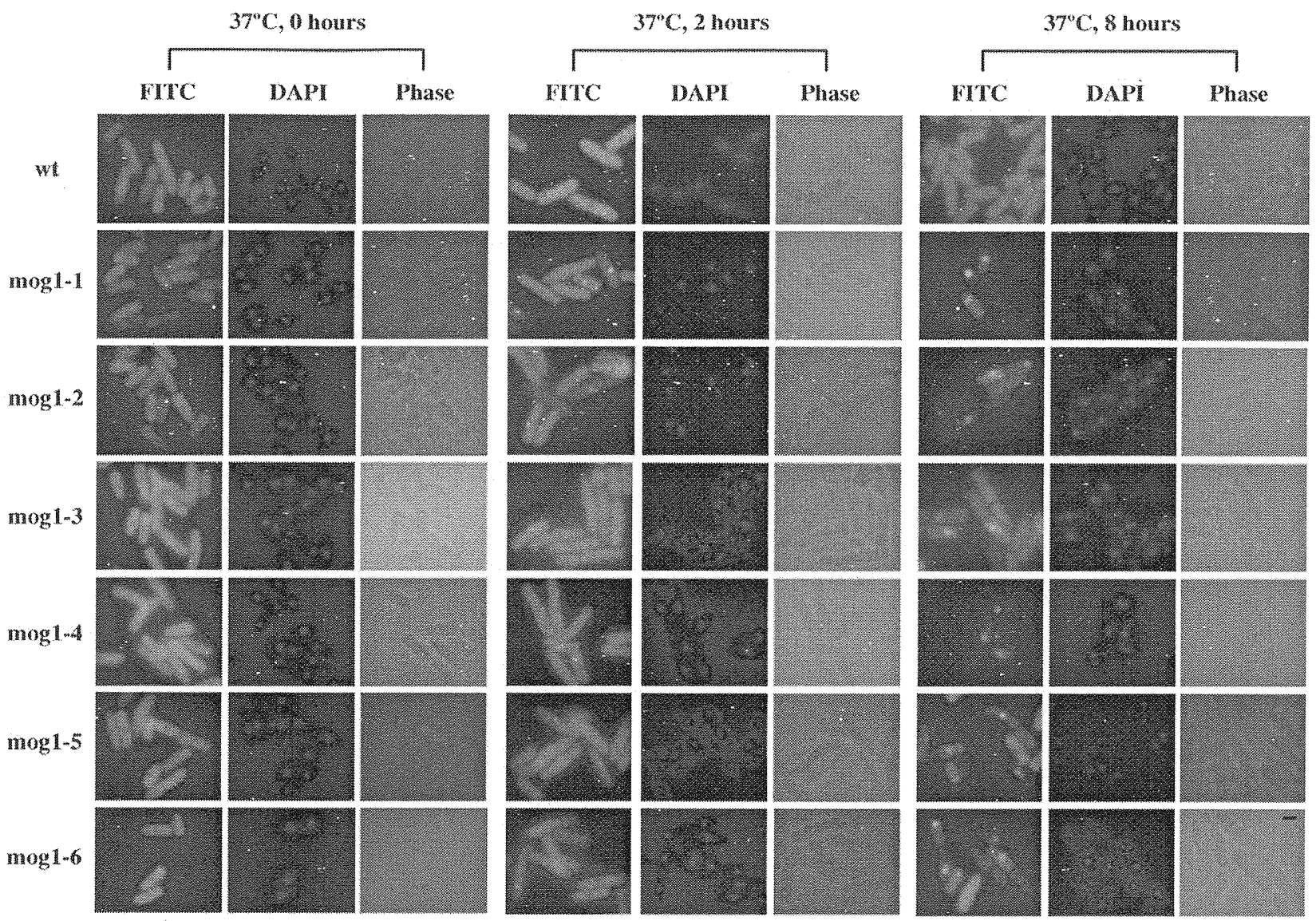

Fig. 5. mRNA export assay. Spmog $l^{\mathrm{wt}}$ and Spmog $I^{\mathrm{ts}}$ were cultured in YES medium at $26^{\circ} \mathrm{C}$ until OD 600 in $=0.2$, and the half of cultures were incubated at $37{ }^{\circ} \mathrm{C}$ (see Fig. 3). Cells were fixed at the indicated time and hybridized with biotin-labeled oligo $(\mathrm{dT})_{49}$, followed by incubation with FITC-avidin. Cellular DNA was stained with DAPI. FITC and DAPI of the same fields of cells are shown, together with the corresponding phase contrast views. Bar: $2 \mu \mathrm{m}$.

Scmogl 1 shows a normal mRNA export (Oki and Nishimoto, 1998), but Spmog 1-1 $1^{\text {ts }}$ has a defect in mRNA export (Tatebayashi et al., 2001). To clarify this issue, mRNA export of all five Spmog $I^{\text {ts }}$ mutants was examined as shown in Fig. 5. The frequency of cells showing the nuclear accumulation of poly (A) RNA was calculated in Table 3. These data indicated that all of Spmog $I^{\text {ts }}$ mutants clearly showed mRNA export defect after $2 \mathrm{~h}$ incubation at $37^{\circ} \mathrm{C}$ when they were still growing (Fig. 3).

\subsection{Nxt2 and Cid13 were co-immunoprecipitated with SpMogl}

The next question is whether suppressors of Spmog $I^{\text {ts }}$ directly interact with SpMogl or not. To address this issue, obtained suppressor genes and Spmogl ${ }^{+}$were tagged with Myc and HA (hemagglutinin), respectively. Myc-tagged suppressor genes and HA-tagged SpmogI $I^{+}$were simultaneously introduced into Spmog $I^{\text {wt }}$. After expression of the introduced genes in the absence of thiamine, HA-SpMog1 was pulled down using the mAb to HA. Proteins that were coimmunoprecipitated with HA-SpMog1, were analyzed by im- munoblotting with the mAb to Myc. Myc-tagged Spil and antiGST antibody were used as a positive and negative control. As reported for $S$. cerevisiae Ran Gsplp (Oki and Nishimoto, 1998), S. pombe Ran Spil was co-immunoprecipitated with SpMog1. Under the same condition, both Nxt2 and Cid13 were apparently co-immunoprecipitated with SpMog1 (lane 3),

Table 3

The frequency of cells (\%) showing the nuclear accumulation of poly (A) RNA

\begin{tabular}{|c|c|c|c|}
\hline \multirow[b]{2}{*}{ Spmog $I^{\text {ts }}$} & \multicolumn{3}{|c|}{ Time ${ }^{t}$} \\
\hline & $0 \mathrm{~h}$ & $2 \mathrm{~h}$ & $8 \mathrm{~h}$ \\
\hline$w t$ & 0.0 & 0.0 & 0.6 \\
\hline Spmog $1-l^{\mathrm{ts}}$ & 2.4 & 92.1 & 64.4 \\
\hline Spmog $1-2^{\mathrm{ts}}$ & 0.6 & 79.2 & 73.6 \\
\hline Spmog $1-3^{\text {ts }}$ & 0.0 & 24.2 & 76.0 \\
\hline Spmog $1-4^{t s}$ & 1.9 & 70.3 & 74.1 \\
\hline Spmog $1-5^{\text {ts }}$ & 4.6 & 58.2 & 65.5 \\
\hline Spmogl-6 $6^{\mathrm{s}}$ & 3.4 & 69.9 & 37.1 \\
\hline
\end{tabular}

After shift up to $37^{\circ} \mathrm{C}$.

$\%$ : more than 150 cells were examined for the nuclear accumulation of poly $(\mathrm{A})$ RNA. 


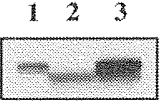

123

123

Anti-Myc

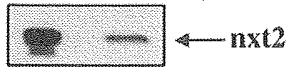

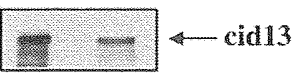

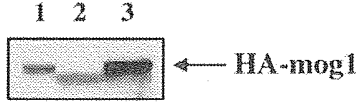

123

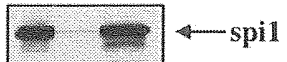

Fig. 6. Mog1 interacted with Nxt2, Cid13 and Spil. HA-Mog1 carried on the vector, pRHA42, and the suppressors, Myc-Nxt2, Myc-Cid13 and Myc-Spil carried on the vector, pRMH41, were co-expressed in Spmogl ${ }^{+}\left(h^{+}\right.$ade6 m-216 leul-32 ura4-418) (Kusano et al., 2004) in medium lacking thiamine. Exponentially growing cells were collected and cell-lysates were prepared as described (Nishijima et al., 2006). From resulting cell-lysates, HA-Mog1 was pulled down using anti-HA antibody (clone HA-7). Proteins co-immunoprecipitated with HA-Mogl were separated with SDS-PAGE and analyzed by immunoblotting with the mAb to Myc (clone 9E10). 1. Total lysates; 2. Pulled down with anti-GST-antibody as a control; 3. Pulled down with the mAb to HA.

compared with the total lysates (lane 1) and the fraction precipitated by the anti-GST antibody (lane 2)(see Fig. 6).

\section{Discussion}

Opi3p is a phospholipid methyltransferase required for the synthesis of phosphatidylcholine that is a major phospholipid constituent of eukaryotic membrane (Boumann et al., 2004). The finding that overexpression of Opi3p rescued Scmog 1A, thus, suggests that Moglp is involved in membrane formation. Consistently, lack of $M O G I$ seems to cause membrane fragile since the cellular body of Scmogl 1 was enlarged (Oki and Nishimoto, 1998) and the nuclear envelope of Spmog $1-I^{\text {ts }}$ was reported to be abnormal (Tatebayashi et al., 2001). Opi3p that is localized in the nuclear periphery, might contribute to strength cellular membrane through the synthesis of phosphatidylcholine. How MOGl is involved in membrane formation is an intriguing future question. Our present result indicated that ScMoglp is required for a membrane localization of Opi3p.

Nxt2 is most homologous to Ntf2p among $S$. pombe Nxt proteins; Nxt1, Nxt2 and Nxt3, all of which possess the Ntf2p domain (Black et al., 1999; BLAST Alignment for S. cerevisiae NTF2 vs S. pombe HITS). The fact that only Nxt2 out of the Nxt family was obtained as a suppressor of Spmog $1^{\text {ts }}$ even with the same frequency as SpMog1, reveals a tight functional relationship of Mog1p with Ntf2p. Indeed, SpMog1 was coprecipitated with Nxt2. Moglp is structurally similar to Ntf2p that make a dimer to transport RanGDP into the nucleus (Stewart and Baker, 2000; Chaillan-Huntington et al., 2001). Since the dimer formation of $\mathrm{Ntf} 2 \mathrm{p}$ depends on the Ntf domain, Ntf $2 p$ is suggested to make a heterodimer with Tapl and Nxt1 that contains a domain homologus to Ntf2p (ChaillanHuntington et al., 2001). In this regard, Moglp could make a heterodimer with $\mathrm{Ntf2p}$ as suggested by the fact that SpMog1 was co-immunoprecipitated with $\mathrm{Nx} 2$. Since $N T F 2$ rescued ScmoglA, a heterodimer of NTF2 and MOG1 might be substituted by a homodimer of NTF2. Whether SpMog 1 could be co-precipitated with $\mathrm{Nxt} 1$ or Nxt3 remains to be investigated.

The other suppressors that were obtained with a low frequency, apparently rescued $\operatorname{Spmog} I^{\text {ts }}$. Furthermore, Cid13 was co-immunoprecipitated with $\mathrm{SpMog} 1$, revealing a tight interaction of SpMog1 with Cid13. Since Cid13 is a poly (A) polymerase for suc22 mRNA that encodes a subunit of ribonucleotide reductase, Cid13 is assumed to regulate the S-
M transition (Saitoh et al., 2002). Taken together these reports regarding Cid13 with the previous report that Spmog $1^{\text {ts }}$ is arrested with G2-M DNA content (Tatebayashi et al., 2001), Mog1 may regulate the cell cycle progression through Cid13. To prove this issue, we tried to determine whether the amount of suc22 mRNA was reduced in Spmog $1^{\text {ts }}$. So far, we could not get a clear answer yet. Thus the question of how SpMog1 functionally interacts with Cid13, remains to be investigated. Crp79 functions for mRNA export like Mex67 that interacts with p15/Nxt3 (Thakurta et al., 2002). Thus, it is possible that Crp79 interacts with Mog1 through Nxt3. The fact that both Cid13 and Crp79 were isolated as a suppressor of Spmog $1^{\text {ts }}$, supports the idea that Mogl is involved in mRNA metabolism, as reported previously (Tatebayashi et al., 2001). Indeed, we confirmed all of Spmogl $l^{\text {ts }}$ mutants have a defect in mRNA export.

Ssp1 is a protein kinase involved in stress response pathway (Matsusaka et al., 1995; Rupes et al., 1999). The fact that Ssp1 suppressed Spmog $I^{\text {ts }}$, therefore, is consistent with the previous report that SpMog1 is involved in stress response pathway ( $\mathrm{Lu}$ et al., 2004). It remains to be examined whether Crp79, Sspl and the protein encoded by ORF: SPBC354.10 could be coimmunoprecipitated with SpMogl.

In conclusion, our results indicate Mog1 functions not only for nuclear import but also for RNA metabolism and signal transduction.

\section{Acknowledgment}

We thank Dr. P. Russell for strains, Dr. S. Sue for use of his microscope, Dr. T. Tani for mRNA export and Dr. S. Saitoh for advice on poly (A) polymerase. This work was supported by Grant-in-Aid for Specially Promoted Research.

\section{References}

Belhumer, P., et al., 1993. GSP1 and GSP2, genetic suppressors of the prp20-1 mutant in Saccharomyces cerevisiae; GTP-binding proteins involved in the maintenance of nuclear organization. Mol. Cell Biol. 13, $2152-2161$.

Black, B., Levesque, L., Holaska, J.M., Wood, T.C., Paschal, B.M., 1999. Identification of an NTF2-related factor that binds Ran-GTP and regulates nuclear protein export. Mol. Cell. Biol. 19, 8516-8621.

Boumann, H.A., Chin, P.T.K., Heck, A.J.R., de Kruijf, B., de Kroon, A.I.P.M., 2004. The yeast phospholipids $N$-methyltransferases catalyzing the synthesis of phospatidylcholine preferentially convert $\mathrm{Di}-\mathrm{Cl}$ 6:1 substrates both in vivo and in vitro. J. Biol. Chem. 279, 40314 40319. 
Carlson, M., Botstein, D., 1982. Two differentially regulated mRNAs with different $5^{\prime}$ ends encode secreted with intracellular forms of yeast invertase. Cell 28, 145-154.

Chaillan-Huntington, C., et al, 2001. NTF2 monomer-dimer equilibrium. J. Mol. Biol. 314, 465-477.

Dasso, M., 2002. The Ran GTPase: theme and variations. Curr. Biol. 12, R502-R508.

Greenberg, M.L., Reiner, B., Henry, S.A., 1982. Regulatory mutations of inositol biosynthesis in yeast: isolation of inositol-excreting mutants. Genetics 100, 19-33.

Hirose, E., et al., 2006. Loss of RanGEF/Pim1 activity abolishes the orchestration of Ran-mediated mitotic cellular events in $S$ pombe. Genes Cells 11, 29-46.

Ifuku, K., Nakatsu, T., Kato, H., Sato, F, 2004. Crystal structure of the PasbP protein of photosystem II from Nicotiana tabacum. EMBO Rep. 5, 362-367.

Kanipes, M.I., Hill, J.E., Henry, S.A., 1998a. The Schizosaccharomyces pombe cho $1+$ gene encodes a phospholipids methytransferase. Genetics 150, 553-562.

Kanipes, M.I., Hill, J.E., Henry, S.A., 1998b. The Schizosaccharomyces pombe chol+ gene encodes a phospholipid methyltransferase. Genetics 150, 553-562.

Kodaki, T., Hosaka, K., Nikawa, J., Yamashita, S., 1991. Identification of the upstream activation sequences responsible for the expression of the phosphatidylethanolamine methylation pathway in Saccharomyces cerevisiae. J. Biochem. 109, 276-287.

Kusano, A., Yoshioka, T., Nishijima, H., Nishitani, H., Nishimoto, T., 2004. Schizosaccharomyces pombe RanGAP homolog, SpRnal, is required for centromeric silencing and chromosome segregation. Mol. Biol. Cell 15, 4960-4970.

Lu, J., M-Y., Deschenes, R.J., Fassler, J.S., 2004. Role for the Ran Binding Protein, Moglp, in Saccharomyces cerevisiae SLN1-SKN7 Signal transduction. Eukaryotic Cell 3, 1544-1556.

Marfatia, K.A., Harreman, M.T., Fanara, P., Vertino, P.M., Corbett, A.H., 2001. Identification and characterization of the human MOG1 gene. Gene 266, $45-56$.

Matsumoto, T., Beach, D., 1991. Premature initiation of mitosis in yeast lacking RCCl or an interacting GTPase. Cell 66, 347-360.

Matsusaka, T., Hirata, D., Yanagida, M., Toda, T., 1995. A novel protein kinase gene $\operatorname{spp} 1+$ is required for alteration of growth polarity and actin localization in fission yeast. EMBO J. 14, 3325-3338.

Mattaj, I., 2004. Sorting out the nuclear envelope from the endoplasmic reticulum. Nat. Rev., Mol. Cell Biol. 5, 65-69.

Maundrell, K., 1993. Thiamine-repressible expression vectors pREP and pRIP for fission yeast. Gene 123, 127-130.

Moore, M.S., Blobel, G., 1994. Purification of Ran-interacting protein that is required for protein import into the nucleus. Proc. Natl. Acad. Sci. U. S. A. 91, 10212-10216.

Nishijima, H., et al., 2006. Nuclear RanGAP is required for the beterochromatin assembly and is reciprocally regulated by histone $\mathrm{H} 3$ and $\mathrm{Clr} 4$ bistone methyltransferase in Schizosaccharomyces pombe. Mol. Biol. Cell 17, $2524-2536$.
Nicolas, F.J., Moore, W.I., Zhang, C., Clarke, P.R., 2001. Xmog1, a nuclear Ran-binding protein in Xenopus, is a functional homologue of Schizosaccharomyces pombe Moglp that co-operates with RanBP1 to control generation of Ran-RanGTP. J. Cell Sci. 114, 3013-3023.

Oki, M., Nishimoto, T., 1998. A protein required for nuclear import, Moglp, directly interacts with GTP-Gsp1p, the Saccharomyces cerevisiae Ran homologue. Proc. Natl. Acad. Sci. U. S. A. 95, 15388-15393.

Oki, M., Nishimoto, T., 2000. Yrblp interaction with the Gsplp C terminus blocks Mog1p stimulation of GTP release from Gsp1p. J. Biol. Chem. 275, 32894-32900.

Oki, M., Noguchi, E., Hayashi, N., Nishimoto, T., 1998. Nuclear protein import, but not mRNA export, is defective in all of the temperature-sensitive mutants of the Saccharomyces cerevisiae Ran homologue, Gsp1-GTPase. Mol. Gen. Genet. 257, 624-634.

Paschal, B.M., Gerace, L., 1995. Identification of NTF2, a cytosolic factor for nuclear import that interacts with nuclear pore complex protein $\mathrm{p} 62 . \mathrm{J}$. Cell Biol. 129, 925-937.

Ribbeck, K., Lipowsky, G., Kent, H.M., Stewart, M., Gorlich, D., 1998. NTF2 mediates nuclear import of Ran. EMBO J. 17, 6587-6598.

Rupes, I., Jia, Z., Young, P.G., 1999. Sspl promotes action depolymerization and is involved in stress response and new end take-off control in fission yeast. Mol. Biol. Cell 10, 1495-1510.

Saitoh, S., et al., 2002. Cid13 is a cytoplasmic poly (A) polymerase that regulates ribonucleotide reductase mRNA. Cell 109, 563-573.

Smith, A., Brownawall, A., Macara, I.G., 1998. Nuclear Import of Ran is mediated by the transport factor NTF2. Curr. Biol. 8, 1376-1386.

Steggerda, S.M., Paschal, B.M., 2000. The mammalian Mog1 protein is a guanine nucleotide release factor for Ran. J. Biol. Chem. 275, 23175-23180.

Stewart, M., 2000. Mechanism of Nuclear Trafficking using Nuclear Transport Factor 2 (NTF2). Cell Struct. Funct. 25, 217-225.

Stewart, M., Baker, R.P., 2000. 1.9 resolution crystal structure of the Saccharomyces cerevisiae Ran-binding protein Mog1p. J. Mol. Biol. 299, 213-223.

Tani, T., Derby, R.J., Hiraoka, Y., Spector, D.L., 1996. Nucleolar accumulation of poly $(A)+$ RNA in heat-shocked yeast cells: implication of nucleolar involvement in mRNA transport. Mol. Biol. Cell 7, 173-192.

Tatebayashi, K., Tani, T., Ikeda, H., 2001. Fission yeast Mog $1 \mathrm{p}$ homologue, which interacts with the small GTPase Ran, is required for mitosis-to-interphase transition and poly(A) RNA metabolism. Genetics 157, 1512-1522.

Thakurta, A.G. et al., 2002. Crp79p, like Mex67p, is an auxiliary mRNA export factor in Schizosaccharomyces pombe. Mol. Biol. Cell 13, 2571-2584.

$\mathrm{Xu}, \mathrm{Q}$, et al., 2006. Crystal structure of an ORFan protein (TM1622) from thermotoga maritime at 1.75 A resolution reveals a fold similar to the Ranbinding protein Mog1p. PROTEINS 65, 777-782.

Wcis, K., 2003. Regulating access to the genome: nucleocytoplasmic transport throughout the cell cycle. Cell 112, 441-451.

Yamada, M., Mattaj, I., Yoneda, Y., 2004. An ATP-dependent Activity that release RanGDP from NTF2. J. Biol. Chem. 279, 36228-36234. 